\title{
DETERMINATION OF SURFACE ROUGHNESS BY GRAZING INCIDENCE X-RAY REFLECTIVITY
}

\author{
D. ŻYMIERSKA \\ Institute of Physics, Polish Academy of Sciences \\ Al. Lotników 32/46, 02-668 Warszawa, Poland
}

\begin{abstract}
The paper presents theoretical calculations of grazing incidence X-ray reflectivity curves for iron and nickel crystals. The computer simulations based on Fresnel theory take into account damping (as the result of surface roughness), which is different from the usual Rayleigh damping. The calculations are performed for a wide range of wavelengths and roughness and also for different distribution of roughness on the surface.

PACS numbers: $61.10 . \mathrm{Dp}, 68.35 . \mathrm{Bs}, 78.20 . \mathrm{Ci}$
\end{abstract}

\section{Introduction}

In recent years the role of surface phenomena in determining the structural, mechanical and magnetic properties of very thin films and multilayers has received an increasing attention. The progress in this field is made mainly due to a great improvement in production and characterization of thin films. X-ray reflectivity is one of the best, nondestructive and high sensitivity tools for studying surface and interface structure on microscopic length scale. The measurement of X-ray reflectivity around the critical angle for total external reflection makes possible accurate determination of thin film thickness and interface roughness, irrespective of the crystalline structure, because the critical angle and the shape of the measured curve are determined essentially by the electron density and are otherwise independent of the amorphous or crystalline structure, or of the orientation of crystallites on/in the surface. Thus, the grazing incidence X-ray reflectometry (GIXR) is equally well applicable to crystalline, polycrystalline, and amorphous materials. The method can be used with conventional X-ray tubes or synchrotron radiation; the latter makes possible to maximize the sensitivity of the method.

$\mathrm{X}$-ray reflectivity studies of the solid surface structure date back to the classic work of Parratt [1], who presented the method of X-ray total reflection for the investigation of surface properties of thick copper film evaporated on a polished glass substrate. Recently, with the advent of synchrotron radiation, this method has attracted much attention. The advantages and disadvantages of neutron and $\mathrm{X}$-rays for reflectivity studies have been discussed by Sinha [2]. Some investigations have been done up to now, e.g. by Sinha et al. [3], Weber and Lengeler [4], Tanner and co-workers [5, 6] and Sakata et al. [7]. 


\section{Total reflection of X-rays from a smooth flat surface}

Crossing an interface between two media with indices of refraction $n_{1}$ and $n_{2}$ (see Fig. 1) X-rays are refracted according to Snell's law

$$
n_{1} \cos \theta_{1}=n_{2} \cos \theta_{2} \text {. }
$$

In condensed matter the index of refraction for hard X-rays is slightly smaller than 1 and can be written as $n=1-\delta-\mathrm{i} \beta$, where $\delta$ and $\beta$ are positive and of the order $10^{-5}$ to $10^{-7}$. In the case considered the X-rays are refracted away from the normal while entering an investigated sample, therefore $\theta_{2}$ will become zero at a finite value of incidence angle $\theta_{1}$ called the critical angle $\theta_{c}$ and given by formula $\theta_{c}=(2 \delta)^{0.5}$ when the absorption is neglected $\left(\beta_{2}=0\right)$. The critical angle $\theta_{c}$ is proportional to the wavelength of radiation and to the square root of the mass density. Below this angle, total external reflection will occur. In condensed matter the critical angle for hard X-rays is below $0.5^{\circ}$.

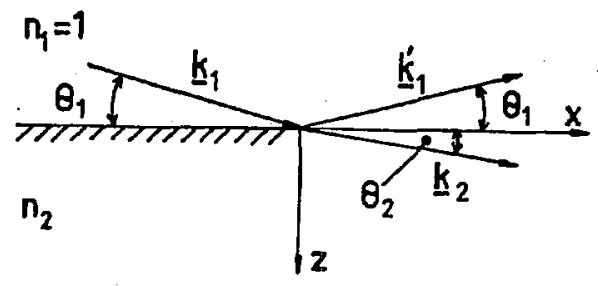

Fig. 1. Refraction and reflection of X-rays at an interface between a vacuum and a solid.

The considerations lead to the Fresnel equations for the reflected and transmitted amplitudes and intensities. In Fig. 1 the reflected beam is indicated by prime, and the incident and the transmitted beams by indices 1 and 2, respectively, since they propagate in media 1 and 2. For small angles (below $1^{\circ}$ ), $\sin \theta$ and $\tan \theta$ can be approximated by $\theta$, and in this case $s$ - and $p$-polarized waves give the same result.

The reflectivity $R$ and transmissivity $T$ are given by

$$
R=\left|\frac{A_{1}^{\prime}}{A_{1}}\right|^{2}=\left|\frac{\theta_{1}-\theta_{2}}{\theta_{1}+\theta_{2}}\right|^{2}, \quad T=\left|\frac{A_{2}}{A_{1}}\right|^{2}=\left|\frac{2 \theta_{1}}{\theta_{1}+\theta_{2}}\right|^{2}
$$

provided that

$$
R+T\left[\operatorname{Re}\left(\theta_{2}\right)\right] / \theta_{1}=1,
$$

as the result of energy conservation.

From the refraction law (Eq. (1)) we have $\theta_{2}=\left(\theta_{1}^{2}-2 \delta_{2}-2 \mathrm{i} \beta_{2}\right)^{0.5}$ so $\theta_{2}$ is complex. Without absorption reflectivity $R$ becomes 1 below $\theta_{c}$ and drops to zero for $\theta_{1}=1.5 \theta_{c}$ according to

$$
R=\delta_{2} /\left(4 \theta_{1}^{4}\right) \text {. }
$$

The absorption drastically reduces the reflectivity below $\theta_{c}$ as it was shown by Stanglmeier et al. [8]. 


\section{Reflectivity from a rough surface}

The surface roughness is a very important factor determining the quality of a crystal surface. The Rayleigh criterion allows one to distinguish between a smooth surface for which $k_{z} \sigma \leq 1$, and a rough surface for which $k_{z} \sigma>1, k_{z}$ being wave vector transfer of the radiation perpendicular to the surface and $\sigma^{2}-$ variance of the height distribution of the surface. 'Consequently, the range of roughness which can be examined depends on the wavelength of the radiation; X-rays as a tool are on the scale between that of the optical microscope and of the electron microscope.

The roughness reduces the reflected amplitude, therefore a damping factor describing an influence of it has to be introduced into the Fresnel equations. The well known Gaussian damping given by Rayleigh is the most widely used roughness correction to the Fresnel reflectivity, although it does not describe well the measured reflectivities of X-rays. If a surface roughness is small (below $5 \mathrm{~nm}$ ) and if the Gaussian distribution of roughness heights is assumed, the reflectivity can be written as

$$
R_{r}=R_{i} \exp \left(-4 k_{1}^{2} \theta_{1}^{2} \sigma^{2}\right)
$$

where $R_{i}$ is the reflectivity from a smooth surface given by Eq.(2), $k_{1}$ is the wave vector of the radiation equal to $2 \pi / \lambda$ with $\lambda$ being the vacuum wavelength and $\sigma$ is a standard deviation.

Since the reflectivities $R_{r}$ and $R_{i}$ in Eq. (5) relate the amplitude of the reflected wave to that of an incident wave, the quantity actually measured in an experiment is $\left|R_{r}\right|^{2}$.

Sakata et al. [7] measured the intensity profile of specular reflected X-rays as a function of scattering angle and determined a verage roughness of polished silicon single crystal surfaces using the formula similar to Eq. (5); it gave no satisfactory profile fits of the experimental data. In general, the experimental and theoretical curves are in quantitative but not in detailed agreement.

There is a possibility to treat the surface roughness as an additional layer of reduced density and of a certain thickness as it was done by Stanglmeier et al. [8]. Also Pynn [9] presented neutron reflectometry as a technique for probing the density of matter close to the solid or liquid surfaces.

\section{Simulated X-ray reflectivity curves}

The aim of this paper is to calculate $\mathrm{X}$-ray reflectivity curves at grazing angle of incidence for different wavelength beams for iron and nickel crystals with a different surface roughness.

The present calculations are based on the conception of a self-affine surface defined by Mandelbrot [10] and adapted to the description of a rough surface by Sinha et al. [3]. The surface roughness can be characterized by a height-height correlation function

$$
C(t)=\sigma^{2} \exp \left[-(t / L)^{2 h}\right]
$$

where $\sigma$ is the root-mean-square value of the surface roughness, $t-$ a distance between two points in the $x, y$ plane for which the height-height correlation is considered, $L-$ a roughness correlation length and $h-$ a roughness exponent 
taking values from 0 for a very jagged surface to 1 for smooth hills and valleys at the surface. For $h=1$ the correlation decays like a Gaussian and for $h=0.5$ as an exponential. formula

Additionally the surface structure factor is used in calculations, given by

$$
\begin{aligned}
S= & \left|q_{z}\right|^{-2} \exp \left\{-0.5 \sigma^{2}\left[\left(q_{z}\right)^{2}+\left(q_{z}^{*}\right)^{2}\right]\right\} \\
& \times \int_{0}^{\infty} \mathrm{d} t \cos \left(q_{r} t\right)\left\{\exp \left[\left|q_{z}\right|^{2} \sigma^{2} C(t)\right]-1\right\}
\end{aligned}
$$

with

$$
q_{r}=0.5 k_{1}\left(\theta_{1}^{2}-{\theta^{\prime}}_{1}^{2}\right) \text { and } q_{z}=k_{1}\left(\theta_{2}+\tilde{\theta}_{2}\right),
$$

where $q$ is the scattering vector.

In the limit $q_{z} \ll 1$ the surface structure factor $S$ reduces to the Fourier transform of the height-height correlation function of the surface given by formula (6).

In the present paper calculations have been performed for iron and nickel surfaces. Parameters concerning the surface such as height of the surface roughness $\sigma$, the correlation length $L$ and the value of $h$ have been chosen in a wide range. Simulations have been done for several different wavelengths. The comparison between four X-ray sources ( $\mathrm{Cr} K_{\alpha}, \mathrm{Cu} K_{\alpha}, \mathrm{Mo} K_{\alpha}$ and $\mathrm{Ag} K_{\alpha}$ with $\lambda$ being equal to $0.2289 \mathrm{~nm}, 0.1541 \mathrm{~nm}, 0.0709 \mathrm{~nm}$ and $0.0551 \mathrm{~nm}$, respectively) is shown in Fig. 2a for iron and in Fig. 2b for nickel; the dependence of the reflectivity on the wavelength of the radiation is evident.
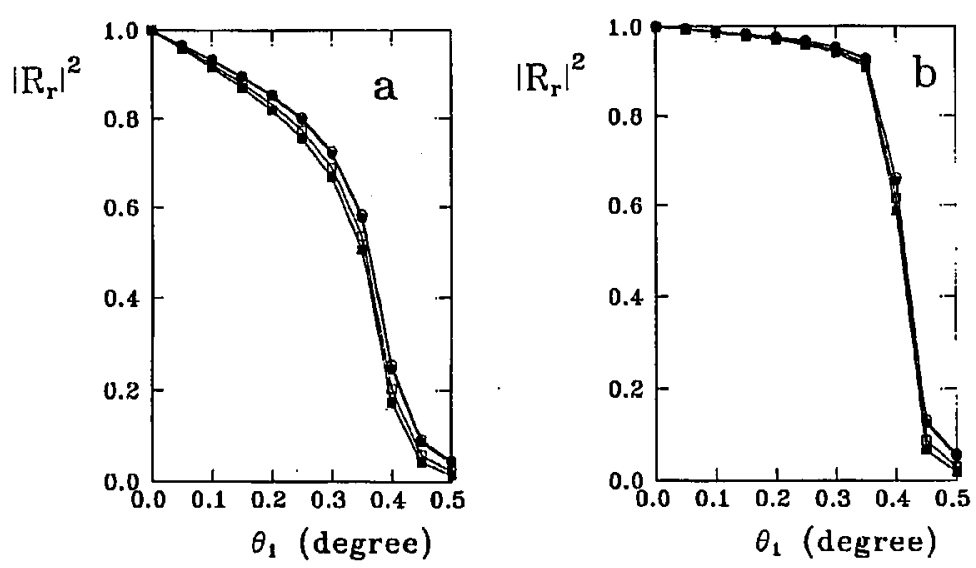

Fig. 2. Grazing incidence X-ray reflectivity curves from iron (a) and nickel (b) surfaces for different radiation beams: $\mathrm{Cr} K_{\alpha}$ (white circle), Cu $K_{\alpha}$ (full circle), Mo $K_{\alpha}$ (white square) and $\mathrm{Ag} K_{\alpha}$ (full square). The surface parameters are $\sigma=0.7 \mathrm{~nm}, L=700 \mathrm{~nm}$, and $h=0.7$ (between a Gaussian and an exponential correlation function decay).

In the ideal case of non-absorbing material with a perfectly flat and smooth surface the grazing incidence $\mathrm{X}$-ray reflectivity falls rapidly above critical angle 

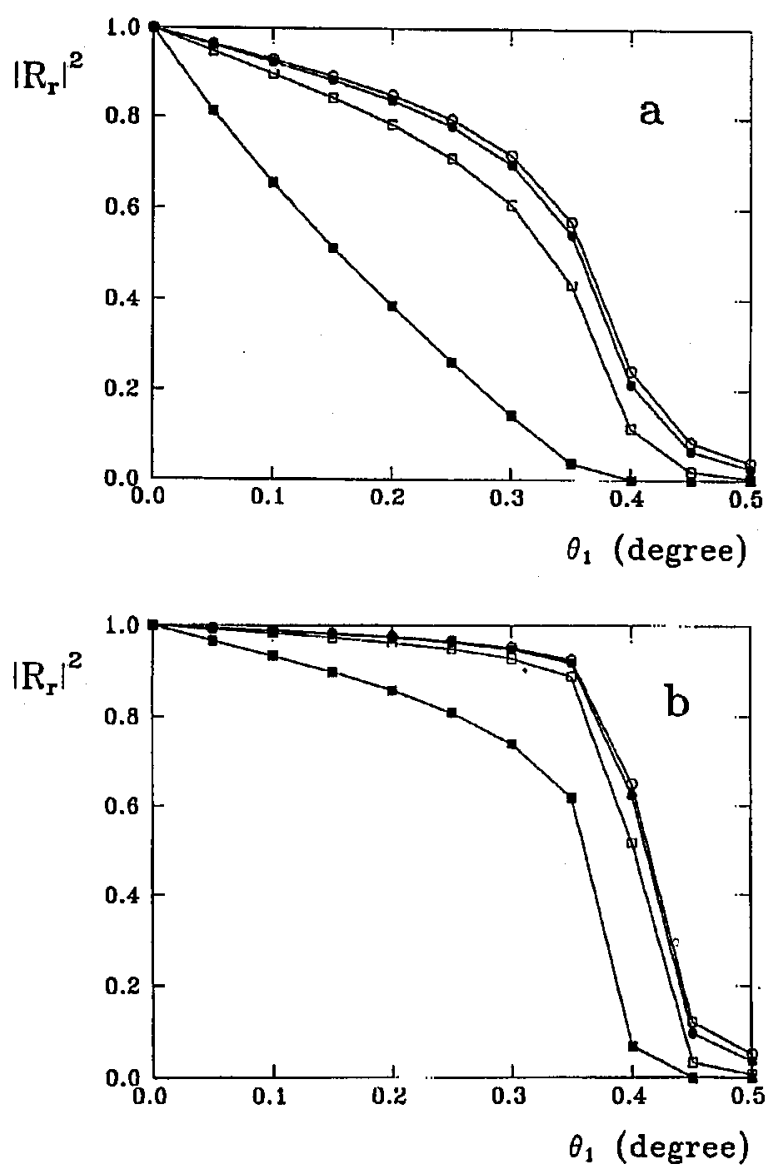

Fig. 3. Simulated reflectivity curves for iron (a) and nickel (b) surfaces with different roughness with $\mathrm{Ag} K_{\alpha}$ radiation. The surface roughness is $\sigma=0.3 \mathrm{~nm}$ (white circle) $\sigma=0.5 \mathrm{~nm}$ (full circle), $\sigma=1.0 \mathrm{~nm}$ (white square) and $\sigma=3.0 \mathrm{~nm}$ (full square).

and this decay is approximately proportional to $\theta_{1}^{-4}$ according to formula (4). As it was expected, surface roughness causes the reflectivity to fall off more rapidly than $\theta_{1}^{-4}$ dependence given for smooth surfaces by formula (4), and this decay is strongly dependent on surface roughness, especially on heights of roughness, and becomes steeper when the height of roughness rises.

In Fig. 3 the reflectivity curves for iron and nickel surfaces with the roughness being as high as $0.3 \mathrm{~nm}, 0.5 \mathrm{~nm}, 1.0 \mathrm{~nm}$ and $3.0 \mathrm{~nm}$ are presented.

\section{Conclusions}

The presented method consists in a comparison between theoretical and observed experimentally X-ray reflectivity curves. By adjusting a theoretical curve to a measured curve, one learns which properties of the surface are responsible for 
the curves observed in experiment, thus the simulated reflectivity curves can be used for interpretation of experimental data.

From the analysis of the X-ray reflectivity curves as a function of such parameters as the height of roughness and the correlation length it follows that the method of grazing incidence X-ray reflectivity allows one to determine the surface roughness of the order of nanometer and to make a distinction between roughness of different heights in this scale (see Fig. 3).

On the other hand, the analysis of the theoretical curves can be useful for choosing the best experimental conditions such as the wavelength of X-ray beam, e.g. in Fig. 2 it is shown that the Ag source is the most suitable for investigations of surfaces of iron and nickel crystals.

\section{Acknowledgment}

The author would like to thank Professor Julian Auleytner of the Institute of Physics of the Polish Academy of Sciences for suggesting the subject of the paper.

This work was partly supported by the Committee for Scientific Research (grant No. 2 PO3B 02 09).

\section{References}

[1] L.G. Parratt, Phys. Rev. 95, 359 (1954).

[2] S.K. Sinha, Physica B 173, 25 (1991).

[3] S.K. Sinha, E.B. Sirota, S. Garoff, H.B. Stanley, Phys. Rev. B 38, 2297 (1988).

[4] W. Weber, B. Lengeler, Phys. Rev. B 46, 7953 (1992).

[5] D.K. Bowen, B.K. Tanner, Nanotechnology 4, 175 (1993).

[6] D.K. Bowen, M. Wormington, Adv. X-Ray Analysis 36, 171 (1993).

[7] O. Sakata, A.Y. Nikulin, H. Hashizume, Jpn. J. Appl. Phys. 32, L616 (1993).

[8] F. Stanglmeier, B. Lengeler, W. Weber, H. Göbel, M. Schuster, Acta Crystallogr. A 48, 626 (1992).

[9] R. Pynn, Phys. Rev. B 45, 602 (1992).

[10] B.B. Mandelbrot, The Fractal Geometry of Nature, Freeman, New York 1982. 